\title{
AN INTERVENTIONAL PROGRAM FOR NURSING STAFF ON SELECTED MASS GATHERING INFECTIOUS DISEASES AT HAJJ
}

\section{By}

\author{
MAMDOUH M. EL-BAHNASAWY ${ }^{1}$, NAGWA ZEIN EL ABDEEN A. ELMENIAWY², \\ and TOSSON A. MORSY ${ }^{3}$
}

Consultant of Endemic Diseases and Fevers, Military Medical Academy ${ }^{1}$, Military Medical Institute, Maadi Military Hospital ${ }^{2}$ and Department of Parasitology, Faculty of Medicine, Ain Shams University, Cairo $11566^{3}$, Egypt

\section{Abstract}

This work improved military nursing staff knowledge on selected mass gathering infectious diseases at Hajj. The results showed that only (20\%) of the participating nurses attended training program about health hazard during pilgrim. But only $(40.0 \%)$ of them found the training programs were specific to nurses. Majority found the program useful $(70.0 \%)$, and the average duration of this training program in weeks was $3.5+1.1$. There was significant improvement $\mathrm{P}=<0.001$, of correct knowledge about meningitis regarding causes, organisms, mode of spread, people at risk, transmission, prevention and treatment, the highest improvement was in causes of meningitis the lowest was in adult vaccination. $25 \%$ of participants had adequate knowledge ( $>60 \%$ from total score) in pre-test $93 \%$ in post-test $72 \%$ after 3 month with significant difference among tests regarding adequate knowledge.

There was significant improvement of correct knowledge $\mathrm{P}=<0.001$ about seasonal influenza and respiratory diseases during pilgrim, the highest improvement was in influenza vaccine strains the lowest was in antiviral drugs. $23 \%$ of nurses had adequate knowledge ( $>60 \%$ from total score) in pre-test $94 \%$ in post-test $66 \%$ after 3 month with significant difference among tests regarding adequate knowledge. There was significant improvement $\mathrm{P}=<0.001$ of correct knowledge about gastrointestinal diseases and food poisoning during pilgrim among nurses at military hospital, the highest improvement was in risk factors of food poisoning the lowest was in what GE patient should do. $22 \%$ of participants had adequate knowledge ( $>60 \%$ from total score) in pretest $91 \%$ in post-test $58 \%$ after 3 month with significant difference among tests regarding adequate knowledge. There was significant improvement $\mathrm{P}=<0.001$ of correct knowledge about heat exhaustion during pilgrim among nurses at military hospital, the highest improvement was in non-communicable diseases the lowest was in sun stroke prevention. $27 \%$ of participant had adequate knowledge ( $>60 \%$ from total score) in the pre-test $94 \%$ in the post-test $74 \%$ after 3 month with significant difference among pre, post and FU regarding adequate knowledge. Also, there were significant improvement $\mathrm{P}=<0.001$ of correct knowledge about hypertension, dengue fever, skin scalding \& others diseases during pilgrim among nurses at military hospital, the highest improvement was in skin scalding prevention the lowest was in first aid bag. $28 \%$ of participant had adequate knowledge ( $>60 \%$ from total score) in the pre-test $92 \%$ in the post-test $61 \%$ after 3 month with significant difference among pre, post and FU regarding adequate knowledge. There was a significant difference between total knowledge score according to education, and work experience $(\mathrm{P}>0.05)$ in the pre, post and after 3 month in age and in all intervention time in department the highest was ICU then ward then operation room.

Key words: Egypt, Military nursing staff, Mass gathering infectious diseases, Pilgrimage

\section{Introduction}

Hajj pilgrimage is a yearly event in which $>2$ million Muslims from around the world gather in Mecca, Saudi Arabia. Such high density of crowding presents a risk for local outbreaks and for worldwide spread of infectious agents (Steffen et al, 2012).
Annually, millions of Muslims embark on a Religious Pilgrimage called the "Hajj" to Mecca in Saudi Arabia. The mass migration during the Hajj is unparalleled in scale, and pilgrims face numerous health hazards. The extreme congestion of people and vehicles during this time amplifies health risks, such as those from infectious diseases, which 
vary each year. Since the Hajj is dictated by the lunar calendar, which is shorter than the Gregorian calendar, it presents public-health policy planners with a moving target, demanding constant preparedness (Ahmed et al, 2006). There are considerable risks for communicable and non-communicable hazards that pilgrims face. With the rise in global travel, preventing disease transmission has become paramount to avoid the spread of infectious disease (Gatrad and Sheikh, 2002).

Religious festivals attract a large number of pilgrims from worldwide and are a potential risk for the transmission of infectious diseases between pilgrims, and to the indigenous population. The threat to global health security posed by infectious diseases with epidemic potential shows the importance of advanced planning of public health surveillance and response at these religious events. Saudi Arabia has extensive experience of providing health care at mass gatherings acquired through decades of managing millions of pilgrims at the Hajj. A mass gathering (MG), as defined by any occasion, either organized or spontaneous, that attracts sufficient numbers of people to strain the planning and response resources of the community, city or nation hosting the event (Memish et al, 2014). MG can be spontaneous or organized and include sports events, social or cultural functions, gatherings of displaced populations due to natural or war, and political or religious congregations (Freedman et al, 2006). In addition to risks of infectious diseases during MG non-communicable risks include the cardiovascular diseases, environment-related heat injury, fire-related injury, illnesses related to use of drugs and alcohol, occupational injuries, trauma or crush injuries associated with stampedes, exacerbation of respiratory diseases, and crowd safety (Alquthami and Pines, 2014). Large MG can also provide opportunities for terrorist activities (Frenk and Chen, 2011). Moreover, there was a substantial amount of over-triage occurred with the existing pre-hospital triage systems, which may lead to misallocation of limited resources (Turris and Lund, 2012).

This study aimed at the development of an intervention program for the Military Nursing Staff on selected mass gathered infectious diseases during travelling to Saudi Arabia

\section{Subjects, Materials and Methods}

The sample consisted of 100 nursing staff who have time to participate in the research and met the inclusion criteria was included in the study.

Rationale: Hajj is an inevitable overcrowding in a confined area of such large numbers from all countries all over the world increases the risk of infection transmitted by close contact and overcrowding. So, the current study will spotlight on selected infectious diseases that could be posing a risk of infection transmission to the Egyptian people via Pilgrims.

Objectives: To assess the Military nursing staff' knowledge as regard the risks of infectious diseases in Mass gathering events such as Hajj before and three months later after program implementation. To determine the Military nursing staff satisfaction towards the intervention program after implementation and to design a simplified illustrative booklet on infectious diseases risks during Hajj

Research design: A quasi-experimental research design with pre-post assessment was used in carrying out this study.

Study setting: The study was conducted in Military Hospital. The hospital provides services to military personnel and their families as well as civilian people.

Inclusion criteria: Nurses who agreed to participate in the emerging educational program, who had at least one year experience and nurses who completed the pre-test at the beginning of the educational program, complete the program and take the post-test and after 3 month post-test. Exclusion criteria include: Pilot study sample was excluded, withdrawal from or inability to complete the educational program, and nursing staff that has legal long leaves.

The instruments used were developed to go on with the Egyptian rules as follows: 
Tool 1: Self-administration Questionnaires: This instrument was divided in to four parts:-

Part1: Demographic Data Questionnaire (DDQ): The DDQ was used to gather data related to age, sex, marital status qualifications, rank, years of experience in nursing, shifts and department. Part2: Knowledge Test (pre/post-test): a- A Questionnaire was developed by the researcher based on review of related literature to collect data related to nursing staff level of knowledge regarding the pilgrim health hazard. b- Nurses' knowledge was evaluated before, immediately after program implementation and 3 months later; through (58) different questions: 1Meningitis during pilgrim (16 items), 2- Influenza \& other respiratory diseases during pilgrim (16 items), 3- GE \& food poisoning (botulism) during pilgrim (11 items), 4- Heat exhaustion during pilgrim (6 items) and 5Hypertension, Dengue fever, skin scalding \& others diseases during pilgrim (9 items)

Scoring system: Each question was evaluated by giving score of "1" for each correct answer, and zero for each wrong answer. These scores were converted into a percent score. The knowledge was considered correct or satisfactory if the percent score was $60 \%$ or more and unsatisfactory if less than $60 \%$.

Tool 2: Educational needs assessment questionnaire: The educational needs assessment questionnaire, consisting of different questions, was developed by the researcher based on review of related literature to determine the unmet educational needs of nursing staff regarding pilgrim health hazard; In addition to identify their previous training and desire to attend an educational program.

Assessment was through the following questions: 1- Health hazard Classification, 2- Meningitis spread and prevention during pilgrim, 3- Respiratory infections during pilgrim, 4- Gastroenteritis and food poisoning during pilgrim, 5- Non-communicable disease during pilgrim, 6- Symptoms \& signs of dependable detection and screening, 7- Prevention \& vaccination, and 8- Infec- tion control. Scoring system: As regards the nurses' opinion about their previous study of the topics; the score of "2" was given for sufficiently studied topics, "1" was given for insufficiently studied topics and zero for non-studied topics. The scores were converted into a percent score. Prioritization of topics was done according to their percent score.

Tool 3: Participants' feed-back form (Program evaluation form): A structured form was developed by the researcher to evaluate the outcome of the program from nursing staff 'point of views. It was include questions related the objectives, time period, content, time schedule of the program and the methods of teaching used immediately after program implementation It was divided into five categories as follow: A- Program Evaluation Dimensions (1- Evaluation of the Tutor, 2- Training materials/ Teaching Aids, 3Lecture duration, 5- Training place, 6- Meeting trainees expectations. B- Evaluate studied topics. C-Scoring system: The evaluation was conducted through giving score of 1 for adequate or satisfied and zero for not for each item.

Designing the educational program by reviewed the accessible up-to-date literature (Textbooks, Scientific Journals and Internet as well as the publications of first and last authors) relevant to the study to formulate the data collection tools prior to the implementation of the educational program.

Educational program objectives were to improve nursing staff knowledge related to pilgrim health hazard. The content of the education program consisted of 8 Sessions, ranged from 30 to 45 minutes for each session. One session: To acquaint the participants about the educational program steps. Six sessions: To improve nursing staff knowledge regarding pilgrim health hazard. One session: To assess nursing staff feedback.

Teaching methods: Group discussion and demonstration and re-demonstration were the main two methods used in the program. Also, different teaching media were used for 
illustration as data show, photos, and video films.

Evaluation of the program: 1- Pre-test: before conducting the program, 2- Post-test: at the end of the program and 3- Follow up (FU) test: 3-months later.

Validity Test: The developed questionnaires were submitted to a panel of experts in the field of nursing education and public health administration for ensuring relevancy, clarity, and readability, ease of understanding, question sequence, and completion time. Then, questionnaires were edited according to experts' suggestions.

A pilot study was carried out on $10 \%$ of the study sample (10 nurses); the aim of the pilot study was to test the applicability of the tools, the feasibility of the study and the time needed for filling the forms. Questionnaires were pre-tested several times to ensure that the wording, format, length, and sequencing of questions were appropriate. During each successive pretest, feedback obtained to help refine quality of the measures. According to the result of this pilot study the tools were assessed and some modification was done. The participants of this pilot study were excluded from the study.

The reliability test was done by using the internal consistency reliability test (Cronbach's alpha). The reliability coefficients were generally high for all questionnaires, and suitable for scientific purposes. The reliability coefficients for each questionnaire as follow: 1- Knowledge test (pre / post-test): 0.82. 2- Educational needs assessment questionnaire: 0.81. 3- Program evaluation form: 0.80

Administrative and ethical design: 1- The study protocol was reviewed and approved by the Military Institute of Health and Epidemiology director. Once approved, an official permission was obtained to conduct the study in the military hospital. 2- Aim of study was clarified to the Director of the Military Hospital and the nursing director of hospital. 3- Informed written consents were taken from each participant before the study. Each nurse was informed about the nature and purpose of the study, and took full right to refuse participation or withdraw at any time. 4- Ensured confidentiality of obtained information.

Data collection procedure: A formal letter from the hospital manager was taken to get his permission to collect data before distributing the questionnaire. The component of the tools was clarified to the manager. He was assured that the collected data would be used for the scientific research only and would be treated with confidentiality. Only code numbers were used to mark sheets.

Before conducting the educational program, the nurses who met the inclusion criteria was identified and asked for participation in this study. The researcher explained to the subjects the purposes, benefits, and ethical considerations of this study. Then the subjects who agreed to participate in this study completed the educational needs questionnaires to collect data related to their previous training and their desire to attend an educational program on pilgrim health hazard. Based on learning needs assessment results, the topics of the program were developed based on the review of related literature.

At the beginning of the implementation phase, pre-test knowledge was evaluated. At the beginning of the first session, an orientation of the program and its purpose took place, and nurses informed about the duration and sessions place. Each session was started by setting the objectives of the new topic. At the end of each session nurses' question was answered. Eight sessions through sixteen meetings formulated our educational program. It was difficult to gather all (100) at one time, so nurses divided into two groups each group consists of (50) and the program was implemented for each group separately for successive (8) weeks. The program was implemented for two days per week (Sunday \& Thursday) one hour per day before starting the morning shift. The duration of each session 
ranged from 8.00 AM to 8.30 AM according to the topics.

At the end of the educational program the Participants' program evaluation Questionnaire was used to evaluate the outcome of the program from Nursing Staff 'point of views. It was included questions related the objectives, time period, content, time schedule of the program and the methods of teaching used. And finally the educational program the nurses' Knowledge Test (post-test) was used at the end of the program and (Follow up-test) 3-months later. Actual fieldwork started at the beginning of February /2012 to the end June / of 2012.

Statistical analysis: Data were presented by tables and graphs, computerized and sta- tistically analyzed using Statistical Package of Social Services version 20 (SPSS), using descriptive statistics in the form of frequencies and percentages for qualitative variables, and means, and standard deviations for quantitative variables. Quantitative continuous data was compared differences between means (quantitative variables) in two groups were compared by Student's t-test multiple groups using one-way analysis of variance test (ANOVA) for multiple group comparisons. Qualitative categorical variables were compared using chi-square. The results were considered significant when p-value $<0.05$ and $\mathrm{p}$-value $<0.01$ and $<0.001$ were considered highly significant (Andow, 2003).

\section{Results}

The results are shown in tables (1 to 11).

Table 1: Socio-demographic and job characteristics of nursing staff at Military Hospital.

\begin{tabular}{|c|c|c|}
\hline Characters & $\mathrm{N}=100$ & $\%$ \\
\hline $\begin{array}{l}\text { Age (years) Mean }(\mathrm{SD}) \\
<25 \\
25-30 \\
>30\end{array}$ & $\begin{array}{l}21.6 \pm 5 \\
64 \\
22 \\
14\end{array}$ & $\begin{array}{l}64 \\
22 \\
14\end{array}$ \\
\hline $\begin{array}{l}\text { Marital status } \\
\qquad \begin{array}{l}\text { Married } \\
\text { Unmarried }\end{array}\end{array}$ & $\begin{array}{l}24 \\
76\end{array}$ & $\begin{array}{l}24 \\
76\end{array}$ \\
\hline $\begin{array}{ll}\text { Rank } & \text { (1) officer } \\
& \text { (2) Solider }\end{array}$ & $\begin{array}{l}20 \\
80\end{array}$ & $\begin{array}{l}20 \\
80\end{array}$ \\
\hline $\begin{array}{l}\text { Graduation } \\
\text { 1.under graduated student } \\
\text { 3. Diploma in Nursing }\end{array}$ & $\begin{array}{l}10 \\
90\end{array}$ & $\begin{array}{l}10 \\
90\end{array}$ \\
\hline $\begin{array}{c}\text { Experience (years) } \quad \text { mean } \pm \text { SD } \\
\qquad 1 \\
1-5 \\
>5\end{array}$ & $\begin{array}{l}3.8 \pm 1.2 \\
50 \\
36 \\
14\end{array}$ & $\begin{array}{l}50 \\
36 \\
14\end{array}$ \\
\hline $\begin{array}{l}\text { Place } \\
\text { 1- wards } \\
\text { 2-operation rooms } \\
\text { 3-ICU }\end{array}$ & $\begin{array}{l}55 \\
25 \\
20\end{array}$ & $\begin{array}{l}55 \\
25 \\
20\end{array}$ \\
\hline $\begin{array}{l}\text { Shift } \\
\text { 1- Morning } \\
\text { 2-Evening } \\
\text { 3- Night }\end{array}$ & $\begin{array}{l}50 \\
32 \\
18\end{array}$ & $\begin{array}{l}50 \\
32 \\
18\end{array}$ \\
\hline
\end{tabular}

Twenty four (24\%) were married, seventy six (76\%) single, with mean age of $21.6 \pm 5,90 \%$ had diploma in nursing but $10 \%$ undergraduate student and $(80 \%)$ of all nurses were soldiers, of them $20 \%$ officers. $50 \%$ were worked less than one year and nurses were worked in wards, operation rooms and
ICU (55\%), (25\%) and (20\%) respectively, $50 \%$ of them working at morning shift $32 \%$ at evening and $18 \%$ at night shift. Educational needs required for implementing an educational program on health hazard in pilgrimage. 
Table 2: Nurses' opinion regarding their previous study of suggested program topics.

\begin{tabular}{|c|c|c|c|c|c|c|c|c|}
\hline \multirow{3}{*}{ Topics } & \multicolumn{4}{|c|}{ Studied } & \multirow{2}{*}{\multicolumn{2}{|c|}{$\begin{array}{l}\text { Not studied } \\
(\text { score }=0)\end{array}$}} & \multirow{3}{*}{$\begin{array}{l}\text { Total } \\
\text { score } \\
(=200)\end{array}$} & \multirow{3}{*}{$\begin{array}{c}\text { Total } \\
\% \\
\text { score }\end{array}$} \\
\hline & \multicolumn{2}{|c|}{$\begin{array}{l}\text { Sufficiently } \\
\text { (score }=2 \text { ) }\end{array}$} & \multicolumn{2}{|c|}{$\begin{array}{l}\text { Insufficiently } \\
\text { (score=1) }\end{array}$} & & & & \\
\hline & No. & $\%$ & No. & $\%$ & No. & $\%$ & & \\
\hline Health hazard classification & 20 & 20.0 & 24 & 24.0 & 56 & 56.0 & 64 & 52.0 \\
\hline Meningitis spread and prevention & 16 & 16.0 & 22 & 22.0 & 62 & 62.0 & 54 & 27.0 \\
\hline Respiratory infections & 18 & 18.0 & 70 & 70.0 & 12 & 12.0 & 106 & 53.0 \\
\hline Gastroenteritis and food poisoning & 30 & 30.0 & 66 & 66.0 & 4 & 4.0 & 126 & 63.0 \\
\hline Non-communicable disease & 6 & 6.0 & 38 & 38.0 & 56 & 56.0 & 50 & 25.0 \\
\hline Symptoms \& signs \& fast detection and screening & 30 & 30.0 & 52 & 52.0 & 18 & 18.0 & 112 & 56.0 \\
\hline Prevention \& vaccination & 16 & 16.0 & 58 & 58.0 & 26 & 26.0 & 90 & 45.0 \\
\hline Infection control & 20 & 20.0 & 40 & 40.0 & 40 & 40.0 & 80 & 40.0 \\
\hline
\end{tabular}

Gastroenteritis and botulism, topics (score $=63 \%$ ); non-communicable one (score $=25 \%$ ).

Table 3: Training history of Nursing Staff.

\begin{tabular}{|c|c|c|c|c|c|}
\hline Nurses' Training history & No & $\%$ & Total & $\chi^{2}$ & $\mathrm{P}$ \\
\hline \multicolumn{3}{|l|}{ Attendance of training program about health hazard } & \multirow{3}{*}{100} & \multirow{3}{*}{$\begin{array}{l}26 . \\
2\end{array}$} & \multirow{3}{*}{$0.001 *$} \\
\hline Yes & 20 & 20.0 & & & \\
\hline No & 80 & 80.0 & & & \\
\hline \multicolumn{3}{|l|}{ Program's specificity to nurses } & \multirow{3}{*}{20} & \multirow{3}{*}{5.0} & \multirow{3}{*}{$0.03 *$} \\
\hline Yes & 8 & 40.0 & & & \\
\hline No & 12 & 60.0 & & & \\
\hline \multicolumn{3}{|l|}{ Nurses' opinion regarding previously attended programs } & \multirow{3}{*}{20} & \multirow{3}{*}{4.0} & \multirow{3}{*}{$0.046^{*}$} \\
\hline Useful & 14 & 70.0 & & & \\
\hline Useless & 6 & 30.0 & & & \\
\hline Average duration of training program (in weeks) & \multicolumn{2}{|c|}{$3.5 \pm 1.1$} & 20 & & \\
\hline
\end{tabular}

* significant at $\mathrm{p}<0.05,20 \%$ attended training program about health hazard. But $40.0 \%$ found programs specific to nurses. $70.0 \%$ found it useful with average duration in weeks $3.5 \pm 1.1$.

Table 4: Main Reason for not attending training program about health hazard during pilgrimage $(\mathrm{n}=80)$

\begin{tabular}{|l|l|l|l|l|l|}
\hline Reasons & No & $\%$ & Total & $\chi^{2}$ test & P value \\
\hline Limited resources & 5 & 6 & & & \multirow{2}{*}{$0.000 * *$} \\
\hline Insufficient time & 40 & 50 & 80 & 27.5 & 0.00 \\
\hline Leaders don't care about nurse training & 30 & 44 & & & \\
\hline
\end{tabular}

**Highly significant at $\mathrm{p}<0.01$, not attending training program insufficient time $(59.5 \%)$.

Table 5: Distribution of participants willing to attend educational program on health hazard during pilgrimage.

\begin{tabular}{|l|l|l|l|l|}
\hline Participants & No & $\%$ & $\mathrm{x}^{2}$ test & P value \\
\hline Willing to attend & 92 & 92.0 & & \multirow{2}{*}{$0.001^{* *}$} \\
\hline Not Willing to attend & 8 & 8.0 & \multirow{2}{*}{84.6} & \\
\hline Total & 100 & 100.0 & & \\
\hline
\end{tabular}

A highly significant difference, $96.0 \%$ willing to attend educational program about health hazard.

Table 6: Main reasons for attendance educational program on health hazard during pilgrimage \& needed topics.

\begin{tabular}{|c|c|c|c|c|}
\hline Main reasons for need of attendance & No. & $\%$ & $x^{2}$ & $\mathrm{P}$ \\
\hline Raise awareness & 44 & 44.0 & \multirow{3}{*}{1.44} & \multirow{3}{*}{0.230} \\
\hline Improve practice & 56 & 56.0 & & \\
\hline Total & 100 & 100.0 & & \\
\hline Needed topics & No. & $\%$ & $\mathrm{x}^{2}$ & $\mathrm{P}$ \\
\hline Meningitis spread and prevention & 16 & 16.0 & \multirow{7}{*}{9.41} & \multirow{7}{*}{0.094} \\
\hline Respiratory infections & 18 & 18.0 & & \\
\hline Gastroenteritis and food poisoning & 24 & 24.0 & & \\
\hline Non-communicable disease & 8 & 8.0 & & \\
\hline Symptoms \& signs \& fast detection and screening & 8 & 8.0 & & \\
\hline Prevention \& vaccination & 26 & 26.0 & & \\
\hline Total & 100 & 100.0 & & \\
\hline
\end{tabular}


Need for attendance to improve practice $(56.0 \%)$ and raise awareness $(44.0 \%)$. Most needed topics prevention \& vaccination (26.0\%), without significant difference.

Table 7: Opinion as regard best duration for educational program to be held.

\begin{tabular}{|l|l|l|l|l|}
\hline Nurses' Opinion Best Duration & No & $\%$ & $\chi^{2}$ & P \\
\hline One Week & 34 & 34.0 & & \\
\hline Two Weeks & 66 & 66.0 & \multirow{2}{*}{10.2} & $0.001^{* *}$ \\
\hline Total & 100 & 100.0 & & \\
\hline
\end{tabular}

**Highly significant at $\mathrm{p}<0.01$, opinion as regard best duration for educational program to be held where $(66.0 \%)$ agreed that best duration for two weeks program.

Table 8: Opinion as regard best time for educational program to be held.

\begin{tabular}{|l|l|l|l|l|}
\hline Nurses' Opinion Best Time & No & $\%$ & $\chi^{2}$ test & P value \\
\hline Morning Shift (8am-2pm) & 50 & 50.0 & & \\
\cline { 1 - 3 } After-Noon Shift (2pm-8pm) & 30 & 30.0 & \multirow{2}{*}{10.5} & \multirow{2}{*}{$0.005^{* *}$} \\
\cline { 1 - 3 } Night Shift (8pm-2am) & 20 & 20.0 & & \\
\hline Total & 100 & 100.0 & & \\
\hline
\end{tabular}

**Highly significant at $\mathrm{p}<0.01$, opinion as regard best time for educational program to be held; where half of participants $(50.0 \%)$ agreed better to at morning shift.

Table 9: Participant's opinion as regard best place for educational program to be held.

\begin{tabular}{|l|l|l|l|l|}
\hline Nurses' Opinion Best Place & No & $\%$ & \multirow{2}{*}{$\chi^{2}$} & P \\
\hline Inside Hospital & 94 & 94.0 & & \\
\hline Outside Hospital & 6 & 6.0 & \multirow{2}{*}{77.4} & $0.001^{* *}$ \\
\cline { 1 - 3 } Total & 100 & 100.0 & & \\
\hline
\end{tabular}

Best place for educational program to be held where (94.0\%) preferred to be inside hospital.

Table 10: Frequency distribution of correct answer about seasonal influenza and respiratory diseases.

\begin{tabular}{|l|l|l|l|l|l|l|l|l|}
\hline \multirow{2}{*}{ Knowledge About } & \multicolumn{2}{|c|}{ Pre answer } & \multicolumn{2}{|c|}{ Post answer } & \multicolumn{2}{c|}{ after 3 month } & \multirow{2}{*}{$\mathrm{X}^{2}$} \\
\cline { 2 - 7 } & $\mathrm{N}=100$ & $\%$ & $\mathrm{~N}=100$ & $\%$ & $\mathrm{~N}=100$ & $\%$ & \\
\hline 1. Commonest Infectious disease & 25 & 25 & 96 & 96 & 55 & 55 & 14.6 \\
2. Lobar pneumonia causative bacteria & 23 & 23 & 95 & 95 & 70 & 70 & 25.6 \\
3. Seasonal influenza patterns & 31 & 31 & 92 & 92 & 75 & 75 & 22.3 \\
4. Antiviral drugs & 36 & 36 & 94 & 94 & 59 & 59 & 12.1 \\
5. People at risk of avian flu & 26 & 26 & 94 & 94 & 62 & 62 & 20.6 \\
6. Influenza vaccine strains & 17 & 17 & 96 & 96 & 64 & 64 & 32.4 \\
7. Classification of influenza pattern & 29 & 29 & 86 & 86 & 54 & 54 & 21.1 \\
8. Factors reduce impacts of respiratory disease & 37 & 37 & 90 & 90 & 79 & 79 & 26.8 \\
9. Prevention of common cold & 19 & 18 & 91 & 91 & 62 & 62 & 24 \\
10. Three viruses of influenza & 30 & 30 & 96 & 96 & 59 & 59 & 19.6 \\
11. Human types & 29 & 29 & 95 & 95 & 72 & 72 & 27.7 \\
12. Animals type & 31 & 31 & 94 & 94 & 74 & 74 & 23.8 \\
13. Avian types & 36 & 36 & 93 & 93 & 65 & 65 & 18.4 \\
14. Avian flu at the past called & 18 & 18 & 94 & 94 & 81 & 81 & 31.9 \\
15. Spread of avian flu among chickens & 30 & 30 & 94 & 94 & 68 & 68 & 23.7 \\
16. Prevention of avian flu & 29 & 29 & 97 & 97 & 73 & 73 & 28.9 \\
Adequate knowledge & 23 & 23 & 94 & 94 & 66 & 66 & 21.3 \\
Total score from (16) & $4.9 \pm 0.9$ & & $14.2 \pm 3.1$ & & $11.9 \pm 2.8$ & & F=14.1 \\
\hline
\end{tabular}

Significant improvement of correct knowledge about seasonal influenza and respiratory diseases, highest improvement in influenza vaccine strains, lowest in antiviral drugs. $23 \%$ had adequate knowledge ( $>60 \%$ from total score) in pre-test $94 \%$ in post-test $66 \%$ after 3 month with significant difference among tests regarding adequate knowledge, also significant difference among tests regarding score average from (16) were $4.9 \pm 0.9,14.2 \pm 3.1$ and $11.9 \pm 2.8$. 
Table 11: Distribution of correct answer on meningitis during pilgrimage regarding causes, organisms, mode of spread, people at risk, transmission, prevention and treatment among nurses.

\begin{tabular}{|l|c|c|c|c|c|c|c|}
\hline Knowledge about & \multicolumn{2}{|c|}{ Pre answer } & \multicolumn{2}{|c|}{ Post answer } & \multicolumn{2}{|c|}{ after 3 month } & \multirow{2}{*}{$\mathrm{X}^{2}$} \\
\cline { 2 - 6 } & $\mathrm{N}=100$ & $\%$ & $\mathrm{~N}=100$ & $\%$ & $\mathrm{~N}=100$ & $\%$ & \\
\hline 1. Infectious disease & 30 & 30 & 96 & 96 & 75 & 75 & 22.6 \\
2. Common causative organisms & 29 & 29 & 95 & 95 & 80 & 80 & 23.6 \\
3. Diagnosis & 31 & 31 & 92 & 92 & 75 & 75 & 39.9 \\
4. Mode and causes of transmission during Umrah \& Hajj & 27 & 27 & 94 & 94 & 72 & 72 & 22.1 \\
5. Definition of meningitis & 26 & 26 & 94 & 94 & 64 & 64 & 20.6 \\
6. Causes & 18 & 18 & 96 & 96 & 83 & 83 & 21.2 \\
7. Modes of transmission & 31 & 31 & 95 & 95 & 65 & 65 & 19.1 \\
8. Aseptic Meningitis most important virus & 36 & 36 & 96 & 96 & 80 & 80 & 24.2 \\
9. Meningitis Vaccine & 26 & 26 & 95 & 95 & 75 & 75 & 22.05 \\
10. Methods of diagnosis & 24 & 24 & 96 & 96 & 80 & 80 & 25.6 \\
11. Meningitis Bacteria names & 29 & 29 & 95 & 95 & 79 & 79 & 23.7 \\
12. Vaccine for adult or 2 years children & 37 & 37 & 94 & 94 & 72 & 72 & 31.7 \\
13. Vaccine safe for infant & 18 & 18 & 93 & 93 & 64 & 64 & 29.2 \\
14. Effectiveness of vaccine beginning & 30 & 30 & 94 & 94 & 84 & 84 & 22.9 \\
15. Time range of effectiveness of vaccine & 29 & 29 & 94 & 94 & 72 & 72 & 23.7 \\
16. The most dangerous type of meningitis & 31 & 31 & 97 & 97 & 74 & 74 & 22.9 \\
Adequate knowledge & 25 & 25 & 93 & 93 & 72 & 72 & 22.8 \\
Total score from (16) & $5.8 \pm 1.1$ & & $14.8 \pm 2.5$ & & $12.4 \pm 41$ & F=13.9 \\
\hline
\end{tabular}

Significant improvement about meningitis items among nurses at military hospital, highest improvement was in causes of meningitis lowest in adult vaccination. $25 \%$ of nurses had adequate knowledge ( $>60 \%$ of total score) in pre-test $93 \%$ in post-test $72 \%$ after 3 month with significant difference among tests regarding adequate knowledge with significant difference among regarding score average from (16) were $5.8 \pm 1.1,14.8 \pm 2.5$ and $12.4 \pm 4.1$.

Table 12: Frequency distribution of correct answer on GI diseases and food poisoning during pilgrimage.

\begin{tabular}{|c|c|c|c|c|c|c|c|}
\hline \multirow[b]{2}{*}{ Knowledge About } & \multicolumn{2}{|c|}{ Pre answer } & \multicolumn{2}{|c|}{ Post answer } & \multicolumn{2}{|c|}{ after 3 month } & \multirow{2}{*}{$\mathrm{X}^{2}$} \\
\hline & $\mathrm{N}=100$ & $\%$ & $\mathrm{~N}=100$ & $\%$ & $\mathrm{~N}=100$ & $\%$ & \\
\hline 1. G.E definition & 17 & 17 & 92 & 92 & 55 & 55 & 23.6 \\
\hline 2. Exhaustion and constipation during G.E & 29 & 29 & 94 & 94 & 80 & 80 & 23.6 \\
\hline 3. What G.E patients should do & 37 & 37 & 94 & 94 & 55 & 55 & 14.3 \\
\hline 4. Prevention & 19 & 19 & 96 & 96 & 79 & 79 & 24.1 \\
\hline 5. Food poisoning definition & 30 & 30 & 85 & 85 & 72 & 72 & 23.6 \\
\hline 6. Chemical Food poisoning & 29 & 29 & 90 & 90 & 64 & 64 & 22.4 \\
\hline 7. Diagnosis & 31 & 31 & 91 & 91 & 74 & 74 & 23.1 \\
\hline 8. Clostridium botulinum & 36 & 36 & 96 & 96 & 68 & 68 & 21 \\
\hline 9. Food poison Risk factor & 18 & 18 & 97 & 97 & 59 & 59 & 24 \\
\hline 10.Food poisoning prevention three principle & 30 & 30 & 94 & 94 & 61 & 61 & 19.6 \\
\hline 11.Staph poisoning & 29 & 29 & 93 & 93 & 42 & 42 & 13.7 \\
\hline Adequate knowledge & 22 & 22 & 91 & 91 & & 58 & 18.7 \\
\hline Total score from (11) & $3.1 \pm 1.3$ & & $9.6 \pm 2.3$ & & $7.7 \pm 2.1$ & & $\mathrm{~F}=12.8$ \\
\hline
\end{tabular}

Table 2: Frequency distribution of correct answer about heat exhaustion during pilgrimage among nurses.

\begin{tabular}{|l|l|l|l|l|l|l|l|}
\hline \multirow{2}{*}{ Knowledge About } & \multicolumn{2}{|c|}{ Pre answer } & \multicolumn{2}{|c|}{ Post answer } & after 3 month & $\mathrm{X}^{2}$ \\
\cline { 2 - 7 } & $\mathrm{N}=100$ & $\%$ & $\mathrm{~N}=100$ & $\%$ & $\mathrm{~N}=100$ & $\%$ & \\
\hline 1. Heat exhaustion definition & 25 & 25 & 96 & 96 & 72 & 72 & 22.6 \\
2. Daily salt need & 23 & 23 & 95 & 95 & 75 & 75 & 23.6 \\
3. Sun stroke sign \& symptom & 31 & 31 & 92 & 92 & 71 & 71 & 21.3 \\
4. Sun stroke Prevention & 36 & 36 & 94 & 94 & 73 & 73 & 20.1 \\
5. Sun stroke TTT & 26 & 26 & 94 & 94 & 74 & 74 & 24.6 \\
6. Non-communicable diseases & 17 & 17 & 96 & 96 & 88 & 88 & 32.4 \\
Adequate knowledge & 27 & 27 & 94 & 94 & 74 & 74 & 23.6 \\
Total score from (6) & $2 \pm 0.4$ & & $5.1 \pm 1.2$ & & $4.2 \pm 1.1$ & & F=8.9 \\
\hline
\end{tabular}

Significant improvement of correct knowledge on heat exhaustion, highest in non-communicable diseases, lowest in sun stroke prevention, $27 \%$ had adequate knowledge ( $>60 \%$ of total score) in pre-test $94 \%$ in post-test $74 \%$ after 3 month with significant difference among tests know-ledge, significant difference among tests regarding score average from (6) were $2 \pm 0.4,5.1 \pm 1.2$ and $4.2 \pm 1.1$. 
Table 3: Distribution of correct answer $(\mathrm{n}=100)$ hypertension, dengue fever, skin scalding \& others diseases.

\begin{tabular}{|l|c|c|c|c|c|c|c|}
\hline \multirow{2}{*}{ Knowledge About } & \multicolumn{2}{|c|}{ Pre answer } & \multicolumn{2}{|c|}{ Post answer } & \multicolumn{2}{|c|}{ after 3 month } & \multirow{2}{*}{$\mathrm{X}^{2}$} \\
\cline { 2 - 6 } & No. & $\%$ & No. & $\%$ & No. & $\%$ & \\
\hline 1. Risk factors of hypertension & 23 & 23 & 96 & 96 & 80 & 80 & 28.6 \\
2. Food \& drinks increase hypertension risks & 28 & 28 & 95 & 95 & 75 & 75 & 23.6 \\
3. Dengue fever characterize by & 31 & 31 & 92 & 92 & 61 & 61 & 21.3 \\
4. Dengue fever vector & 35 & 35 & 94 & 94 & 63 & 63 & 20.4 \\
5. Skin scalding definition & 25 & 25 & 94 & 94 & 74 & 74 & 24.9 \\
6. Skin scalding prevention & 17 & 17 & 96 & 96 & 85 & 85 & 32.4 \\
7. Barber without license may be risk factor & 29 & 29 & 86 & 86 & 72 & 72 & 27.1 \\
8. First aid bag & 37 & 37 & 90 & 90 & 67 & 67 & 20 \\
9. Insulin preservation & 18 & 18 & 91 & 91 & 65 & 65 & 21 \\
10. Adequate knowledge & 28 & 28 & 92 & 92 & 61 & 61 & 20.9 \\
Total score from (9) & $2.8 \pm 0.3$ & & $8.1 \pm 1.8$ & & $6.7 \pm 1.7$ & & F=9.1 \\
\hline
\end{tabular}

Significant improvement about all items, highest improvement in skin scalding prevention, lowest in first aid bag. $28 \%$ had adequate knowledge ( $>60 \%$ of total score) in pre-test $92 \%$ in post-test $61 \%$ after 3 month with significant difference among tests regarding adequate knowledge, significant difference among tests regarding score average from (9) were $2.8 \pm 0.3,8.1 \pm 1.8$ and $6.7 \pm 1.7$.

Table 15: Evaluation of program outcome from nursing staff 'point of views.

\begin{tabular}{|l|c|c|c|c|c|c|}
\hline \multirow{2}{*}{ Dimension } & \multicolumn{2}{|c|}{ Adequate } & \multicolumn{2}{|c|}{ Inadequate } & \multirow{2}{*}{$\mathrm{X}^{2}$} & \multirow{2}{*}{$\mathrm{P}$} \\
\cline { 2 - 7 } & No. & $\%$ & No. & $\%$ & $\mathrm{X}^{2}$ & \\
\hline 1. Evaluation of tutor & 98 & 98.0 & 2 & 2.0 & & \\
2. Training materials/ teaching aids & 91 & 91.0 & 7 & 7.0 & & \\
3. Lecture duration & 89 & 89.0 & 11 & 11.0 & \multirow{2}{*}{14.7} & $0.001^{*}$ \\
4. Place of training & 93 & 93.0 & 7 & 7.0 & & \\
5. Meeting trainees expectations & 96 & 96.0 & 4 & 4.0 & & \\
\hline
\end{tabular}

*significant at $\mathrm{p}<0.05$, evaluation for tutor $(98.0 \%)$, while least for program duration $(82.0 \%)$. Table 4: Evaluation of studied topics from trainee point of view

\begin{tabular}{|l|ll|ll|}
\hline Topics & \multicolumn{2}{|c|}{ Sufficiently } & \multicolumn{2}{|l|}{ Insufficiently } \\
\hline Meningitis & 75 & $75 \%$ & 25 & $25 \%$ \\
\hline Influenza & 86 & $86 \%$ & 14 & $14 \%$ \\
\hline Gastroenteritis & 83 & $83 \%$ & 17 & $17 \%$ \\
\hline URI & 83 & $83 \%$ & 17 & $17 \%$ \\
\hline Non-Communicable & 84 & $84 \%$ & 16 & $16 \%$ \\
\hline Vaccination & 91 & $91 \%$ & 9 & $9 \%$ \\
\hline Prevention & 85 & $85 \%$ & 15 & $15 \%$ \\
\hline
\end{tabular}

Most sufficient studied topic, vaccination and least, meningitis.

Table 5: Relationship between Total knowledge Score \& Nurses' socio-demographic characteristics:

\begin{tabular}{|c|c|c|c|c|c|}
\hline Intervention & \multicolumn{2}{|c|}{ Socio-demographic characteristics } & ANOVA & P-Value \\
\hline Age & $<25$ & $25-30$ & $>30$ & & \\
\hline Pre & $21.25 \pm 12.7$ & $23.45 \pm 10.43$ & $26.33 \pm 5.52$ & 0.335 & 0.571 \\
\hline Post & $85.33 \pm 8.91$ & $90.32 \pm 9.85$ & $96.56 \pm 11.73$ & 3.850 & $0.025^{*}$ \\
\hline After 3 month & $57.31 \pm 12.55$ & $67.23 \pm 10.47$ & $79.54 \pm 11.63$ & 3.9 & $0.017^{*}$ \\
\hline Education & Student & Graduated & & $\mathrm{t}$ & \\
\hline Pre & $24.65 \pm 7.6$ & $25.17 \pm 9.5$ & & 1.8 & 0.08 \\
\hline Post & $90.5 \pm 11.2$ & $95.7 \pm 7.52$ & & 3.263 & $0.02^{*}$ \\
\hline After 3 month & $65.11 \pm 10.93$ & $74.12 \pm 8.62$ & & 0.897 & 0.410 \\
\hline Experience & $<1$ & $1-5$ & $>5$ & $\mathrm{~F}$ & \\
\hline Pre & $23.77 \pm 10.43$ & $26.12 \pm 10.78$ & $28.55 \pm 15.55$ & 2.678 & 0.072 \\
\hline Post & $84.18 \pm 14.93$ & $90.67 \pm 15.46$ & $94.43 \pm 20.5$ & 3.290 & $0.045^{*}$ \\
\hline After 3 month & $62.22 \pm 12.63$ & $65.14 \pm 12.68$ & $66.89 \pm 11.32$ & 2.356 & 0.223 \\
\hline Department & Wards & Operation room & ICU & & \\
\hline Pre & $23.77 \pm 10.43$ & $20.12 \pm 10.78$ & $32.55 \pm 11.55$ & 3.54 & $0.04^{*}$ \\
\hline Post & $88.18 \pm 6.93$ & $82.67 \pm 10.46$ & $95.43 \pm 26.44$ & 3.67 & $0.03^{*}$ \\
\hline After 3 month & $62.22 \pm 12.63$ & $55.14 \pm 12.68$ & $76.89 \pm 11.32$ & 3.8 & $0.03^{*}$ \\
\hline
\end{tabular}


Nurses in age group $>30 \mathrm{Y}$ got highest total knowledge score than others. Graduated nurses got highest total knowledge score; work experience $<5$ years got highest total knowledge score, significant difference between total knowledge score on education, and work experience in pre, post and after 3 month in age and all intervention time in department highest ICU, ward then operation room.

\section{Discussion}

In the present study, the socio-economic status: Twenty four (24\%) were married seventy six $(76 \%)$ were unmarried. the mean age was $21.6 \pm 5.90 \%$ had diploma in nursing but $10 \%$ still undergraduate student and $(80 \%)$ of all nurses were soldiers , $20 \%$ were officers. $50 \%$ of nurses were worked less than one years and nurses were worked in wards, operation rooms and ICU (55\%), $(25 \%)$ and $(20 \%)$ respectively, $50 \%$ of them working at morning shift $32 \%$ at evening and $18 \%$ at night shift. Regarding trainee need assessment and previous training our prior need assessment showed that gastroenteritis and botulism were the most studied topics $($ Total score $=63 \%$ ); because of prevalent of these problem while non-communicable disease during pilgrim were the least topics $($ Total $\%$ score $=25.0 \%)$. This agreed with Al-Shehri and Khoja (2009) who reported that Pre-Hajj health-related advice (diarrhea and gastrointestinal diseases) as most required topic regarding health hazard.

In the present study, only (20\%) attended previous training program about health hazard during pilgrim. But, only (40.0\%) found the training programs were specific to nurses. Moreover $(70.0 \%)$ found the program useful with average duration of $3.5 \pm 1$.1 in weeks, also agreed with Almas et al. (2003). Helminen et al. (2014) found that nursing students felt that they had spent enough time with their mentors during their clinical practice period to ensure that the mentors could assess their behavior. Mentors also evaluated that they had spent enough time with the students. Students and mentors both indicated occasional difficulties with the language used in the competence assessment document. Most of the nursing students and mentors shared the view that it is always necessary for a teacher to be involved in the final assessment discussion. They concluded that nursing students' clinical practice assessment already includes many good practices, but there were still some difficulties in ensuring effective measures of competence.

Regarding the main reason for not attending training program about health hazard during pilgrim was the "insufficient time" (59.5\%), most nurses $(96.0 \%)$ was willing to attend educational program about health hazard during pilgrim. This was to improve practice $(56.0 \%)$ and raise awareness $(44.0 \%)$. The most needed topics were prevention \& vaccination $(26.0 \%)$, without statistically significant difference. Parant et al. (2014) reported that an educational program for pediatric medical-surgical nurses who were expected to staff the pediatric medical-psychiatric unit was developed and implemented prior to the opening of the new unit. Walsh et al. (2014) reported that Although residents recognized nurse practitioners (NPs)' value in team functioning and knowledge in specialized areas, they were unclear about NPs' scope of practice Depending on residents' level of training, residents tended to respond differently to teaching by NPs. More of the senior residents believed they needed to think like physicians and preferred clinical teaching from physician teachers. Junior residents valued the step-by-step instructional approach used by NPs who had a decreased sense of vulnerability when being taught by NPs. Training in teaching skills was helpful for NPs. Barriers to providing optimal education included opportunity, time, and physician attitudes.

Nurses' opinion as regard best duration for educational program to be held where $(66.0 \%)$ of them agreed that the best duration for the program was two weeks, as regard best time for the educational program to be held $(50.0 \%)$ agreed that it was better to be held at morning shift. Regarding best place for the educational program to be held 
where $(94.0 \%)$ of participants preferred that it would be better to be inside hospital. Aarnio et al. (2010) found that when introducing the multidisciplinary subjects to medical students, it is important to think through the clinical relevance of the topic and how it is introduced to medical students. Layne et al. (2010) evaluated the educational model through a focus group session, and found that the morning report was a unique environment where junior doctors feel comfortable engaging with group case-based teaching, with the support and encouragement of senior consultants, reinforced with online case summaries and blog resources. Price (2013) stated that there was widespread recognition that many newly qualified nurses find it difficult to make the transition from completing their university course to taking up their first registered nurse post. He concluded that preceptorship concerns four main areas: orientation to patients and services provided locally, real-time clinical reasoning, skill review and refinement, and socialization within the healthcare team. Parker et al. (2014) explored the psychosocial, educational, and administrative support needed of labor and delivery (L\&D) nurses who care for women undergoing pregnancy termination. They concluded that ensuring continuity of care through knowledge sharing related to genetic counseling and community resources creates the context for holistic patient care. Increased attention to the particular needs of L\&D nurses providing care to women undergoing termination may enhance the quality and safety of care for this unique population.

Meningitis during Hajj, carrier rates for meningococcal disease (MCD) rise to a level as high as $80 \%$ (Al-Gahtani et al, 1995) due to intense overcrowding, high humidity and dense air pollution. When rates of carriage rise to this level, the risk for meningococcal outbreaks becomes a real concern. Memish et al. (2010) in Saudi Arabia stated that an area, in which meningococcal disease outbreaks have frequently occurred, was the African meningi- tis belt, where epidemics of meningococcal disease with a peak incidence as high as $100-800 / 100,000$ population/year have been reported. Another risk factor is mass gatherings including the Islamic pilgrimage to Makkah Al-Mokarama where outbreaks of meningococcal disease have repeatedly occurred. The latest outbreaks occurred during Hajj pilgrimages of 2000 and 2001, when a shift from serogroup A disease to serogroup W135 occurred. Vaccination against serogroups A, C, W-135 and Y with novel conjugate vaccines may help protect individuals and reduce the spread of bacterial carriage and disease. Individuals who should be vaccinated include travelers to epidemic or hyperendemic areas (as identified by international health authorities), travelers for Umra or Hajj (pilgrims), travelers to high risk countries or regions (African meningitis belt) during dry season or countries in subSaharan Africa outside the meningitis belt (where outbreak of meningitis was reported in the preceding 2-3 years), military recruits or deployed military personnel, immunocompromized travelers and high school and college students.

In Egypt, Morsy (2012) stated that often people yearn of a greater travel adventure like a year in Africa to get away from their routine situations and locations, for a quick vacation or as a means of finding one's self, to enjoy new experiences, to learn and experience new languages, new food, and new cultures. While most people associate SubSaharan Africa with safaris, there are endless other possibilities for adventure. Some African countries have actual entry requirements and won't let you in unless you have proof you've been vaccinated against a specific disease. Every African country is unique in the quality of its parks and reserves, roads and other infrastructure, visitor accommodations and receptivity to tourists. Creating an itinerary and arranging travel logistics can intimidate even the most enthusiastic tourist. Others travel more nowadays for the better economy and the advance- 
ments in transportation make it easier to do so. If family members do not reside in the same country or are probably working abroad, other family members would want to visit them and would do so as frequently as their resources would allow. Visiting families and friends in different states or countries are probably the most important reasons for taking time off to travel. No doubt, travelling to different climates, cultures and environments abroad can expose to tropical infectious diseases and health risks. This review dealt with skin lesions in the returning travelers.

In the present study, there were significant improvement of correct knowledge about meningitis during pilgrim regarding causes, organisms, mode of spread, people at risk, transmission, prevention and treatment among nurses at military hospital, the highest improvement was in causes of meningitis the lowest was in vaccine of meningitis of adult. $25 \%$ of nurses had adequate knowledge ( $>60 \%$ from total score) in the pre-test which indicated poor base line knowledge regarding this topic, $93 \%$ in the post-test $72 \%$ after 3 month with significant difference among pre, post and FU regarding adequate knowledge that indicate the effectiveness of the program and good training materials. This data agreed with Memish et al. (2003) who reported significant improvement in knowledge about meningitis during pilgrim specially mode of transmission and causes and they relayed that on special interest of both trainers and trainee toward this topics. Madani and Ghabrah (2007) assessed the compliance of health care workers (HCWs) employed in Hajj in receiving the meningococcal, influenza, and hepatitis B vaccines. They found that the meningococcal and hepatitis $\mathrm{B}$ vaccination coverage level among HCWs in Hajj was suboptimal and the influenza vaccination level was notably low. They added that strategies to improve vaccination coverage among $\mathrm{HCWs}$ should be adopted by all health care facilities in Saudi Arabia. Rajoo et al. (2013) stated that tropical neuroinfections are still cause of substantial mortality in travelers. Therefore, good knowledge of early symptoms is very important for nurses acting as first contact persons. However, nursing students and recent graduates in two $\mathrm{PhD}$ programs demonstrated acceptable, although not large-scaled, knowledge of early signs and symptoms of tropical neuroinfections.

In Egypt, meningitis was encountered in children <6years was $H$. influenzae $(39 \%)$, S. pneumoniae (30\%), N. meningitidis (13\%), Mycobacterium tuberculosis (8\%) and $(10 \%)$ other bacteria (Youssef et al, 2004). Afifi et al. (2007) found the overall case-fatality rate for culture-positive cases was $26 \%$ and was highest among patients with M. tuberculosis (47\%). Factors significantly associated with death $(p<0.05)$ included admission to rural hospitals, long prodromal period, referral from other hospitals, antibiotic treatment prior to admission, and clear CSF $(<100$ cells $/ \mathrm{mm} 3)$.

Upper respiratory tract: Acute respiratory tract infections are very common during the Hajj, particularly so when the pilgrimage falls in the winter season. The close contact among pilgrims during periods of intense congestion, their shared sleeping accommodations (mainly in tents) and the dense air pollution all combine to increase the risk of airborne respiratory disease transmission. A viral etiology of upper respiratory tract infection (URTI) is most commonly implicated at the Hajj but bacterial super-infection often follows. More than 200 viruses can cause URTI but at the Hajj the main culprits are respiratory syncytial virus (RSV), parainfluenza, influenza and adenovirus (Balkhy et al, 2004).

Monne et al. (2008) stated that since early 2007, the Kingdom of Saudi Arabia had experienced several highly pathogenic avian influenza (HPAI) H5N1 outbreaks in the falconry and poultry sectors. The public health threat associated with peculiar husbandry systems, requiring close contact between humans and birds of prey, highlights 
the need of an improved understanding of the epidemiology and of the viral characteristics of $\mathrm{H} 5 \mathrm{~N} 1$ viruses circulating in the region. They identified specific amino acid mutations, which are described as genetic signatures of human influenza A viruses or known to confer resistance to antiviral drugs, raises concerns for the possible human health implications of the KSA H5N1 viruses. Hemida et al. (2014) stated that a prospective study of a dromedary camel herd during the 2013-14 calving season showed Middle East respiratory syndrome coronavirus infection of calves and adults Virus was isolated from the nose and feces but more frequently from the nose. Preexisting neutralizing antibody did not appear to protect against infection.

Memish et al. (2014) reported that MERS$\mathrm{CoV}$ was not the cause of severe CAP in any of the hospitalized pilgrims investigated. They identified a variety of other respiratory pathogens in the sputum of this small number of patients. This indicates that the etiology of severe CAP in Hajj is complex with implications regarding its management.

In Egypt, El-Sayed et al. (2013) stated that in opposite to most countries, avian influenza virus $\mathrm{H} 5 \mathrm{~N} 1$ became endemic in Egypt. Since, its first emerge in 2006 in Egypt, the virus could infect different species of birds and animals and even human. Beside the great economic losses to the local poultry industry in Egypt, the virus infected 166 confirmed human cases, 59 cases ended fatally. They collected sera from human, cattle, buffaloes, sheep, goat, horses, donkeys, swine, sewage rats, stray dogs and stray cats. The sera were collected from Cairo and the surrounding governorates to be examined for the presence of anti-H5N1 antibodies. Clear differences in the seroprevalence were noticed among different species and also between the results obtained by both techniques indicating the difference in test accuracy. They concluded the wide spread of the $\mathrm{H} 5 \mathrm{~N} 1$ virus in the Egyptian environment.

Khalil et al. (2013) suspected the wide spr- ead and re-emerging of acute respiratory syndrome (SARS) coronavirus even to Egypt. Chu et al. (2014) identified near-full-genome sequence $(29,908 \mathrm{nt},>99 \%)$ of Middle East respiratory syndrome coronavirus (MERS$\mathrm{CoV}$ ) from a nasal swab specimen from a dromedary camel in Egypt. They found that viruses genetically very similar to human MERS-CoV are infecting dromedaries beyond the Arabian Peninsula, where human MERS-CoV infections have not yet been detected. These are more or less the main Middle East respiratory syndrome (MERS) as of 9 May 2014, 536 confirmed human cases have been reported to with 145 deaths (WHO, 2014)

In the present study, there were significant $(\mathrm{P}<0.001)$ improvements of knowledge on seasonal influenza and respiratory diseases during pilgrim among nurses at military hospital, the highest improvement was in influenza vaccine strains the lowest was in antiviral drugs. $23 \%$ of participants had adequate knowledge ( $>60 \%$ from total score) in the pre-test which was very poor base line knowledge, $94 \%$ in the post-test $66 \%$ after 3 month with significant difference among pre, post and FU regarding adequate knowledge and that prove the success of our educational program. Sheahan and Musialowski (2001) reported that nurses must consider the many age-related respiratory system changes when assessing and managing respiratory-related symptoms of older individuals. Tromba et al. (2014) stated that it was necessary to implement the educational and informative quality of the triage operators and educators, planning periodical triage training courses to reduce errors. Particular emphasis must be placed on providing pediatric continuing education for nurses practicing in general ED.

Respiratory allergy: Al-Qurashi (2006) in Saudi Arabia, reported that the house dust mites is (HDM) still one of the many causes of allergy, particularly atopic dermatitis. In the houses of children who were suffering allergic manifestations as atopic dermatitis, 
respiratory allergy, bronchitis, skin rash and fever. Double manifestations with fever were reported in four children. In the present study, two types of mites were recovered, Dermatophagoides farinae and Ornithonyssus bacoti. The immunoglobulins as well as complements (C3 \& $\mathrm{C} 4)$ were measured in the patients and cross-matched controls. The complements did not show any significant difference among patients and controls. Elevation was in $\operatorname{IgG}$ and $\operatorname{IgM}$, but not in $\operatorname{IgA}$. High significant elevation was in IgE.

Saleh et al. (2013a) in Egypt stated that HDM can be found worldwide where human beings live independent from the climate and are a major source of multiple allergens. Mite allergens sensitize and induce perennial rhinitis, asthma, or atopic dermatitis in a large portion of patients with allergic disease particularly children. They examined dust from a hospital and the private home of some nursing staff, found seven species of mites. They stated that the presence of mites in and out doors in a hospital and dwellings of medical personnel pave the way to consider HDM as occupational or nosocomial allergens. Saleh et al. (2013b) improved nurses' knowledge and performance regarding the management of HDM to minimize nosocomial patient's exposure in a Military Hospital. All staff nurses with working experience of at least one year were included $(n=60)$. Three tools were used for data collection: 1- a self-administered questionnaire sheet to assess subjects sociodemographic data and knowledge regarding management of HDM, 2- an observation check list to evaluate performance as regard environmental care related to HDMs' control, and 3practical dust collection from indicative areas whenever possible to isolate mites from dust patients' dwelling for identification following standard local and international keys. They found that the implementation of educational intervention program led to their significant improvement of knowledge and performance related to HDM post program implementation. This fact was practically approved as some nurse's requested to examine even their own homes approved as some nurse's requested to examine even their own private homes.

Gastrointestinal diseases: Traveller's diarrhea is common during Hajj, although few studies have documented its incidence and etiology. The last study was in 2002 showed that diarrhea was the third most common cause for hospitalization during Hajj. AlTawfiq and Memish (2012) reported that Hajj would take place during 24-29 October. They added that outbreaks of Ebola hemorrhagic fever in Uganda and the Democratic Republic of the Congo, cholera in Sierra Leone, and infections associated with a novel coronavirus in Saudi Arabia and Qatar required review of the health recommendations of the 2012 Hajj. They gave current guidelines foresee mandatory vaccination with quadrivalent meningococcal vaccine for all pilgrims, yellow fever and poliomyelitis for pilgrims from high-risk countries. Influenza vaccine is strongly recommended.

In the present study, there were significant improvement of correct knowledge about gastrointestinal diseases and food poisoning during pilgrim among the nurses, the highest improvement was in risk factors of food poisoning, and the lowest was in what GE patient should do. A total of $22 \%$ of nurses had adequate knowledge ( $>60 \%$ of total score) in the pre-test $91 \%$ in the post-test $58 \%$ after 3 month with significant difference among 3 tests regarding adequate knowledge that was good indicator for program impact on the trainee knowledge.

Freedman et al. (2008) found that multiple regression analysis revealed the reported number of prior visits for gastroenteritis was inversely associated with overall caregiver score (beta coefficient, $-0.26 ; 95 \%$ confidence interval, $-3.04,-0.28 ; \mathrm{P}=.02)$. Internal consistency was demonstrated with a Cronbach's alpha of 0.67 at time. They concluded that the caregiver gastroenteritis knowledge questionnaire had application in identifying knowledge gaps in gastroenteritis management and measuring improvement following 
educational interventions. Paput (2014) in France stated that improving the daily life of patients suffering from inflammatory bowel disease is one of the objectives of the gastroenterological team of Nice General Hospital. He added that the practice of multidisciplinary team gave nurses the opportunity to fully express their unique role.

Heat exhaustion: When Hajj falls during the summertime temperatures in the Hajj premises may reach from 37 to $45^{\circ} \mathrm{C}$. Generally, Kravchenko et al. (2013) reported that a comprehensive approach to minimize the health effects of extreme heat is required and must address educating the public of the risks and optimizing heat-wave response plans, which included improving access to environmentally controlled public havens, adaptation of social services to address the challenges required during extreme heat, and consistent monitoring of morbidity and mortality during periods of extreme temperatures. Becker and Stewart (2011) stated that once core temperature was $104^{\circ} \mathrm{F}\left(40^{\circ} \mathrm{C}\right)$, cellular damage occurred, initiating a cascade of events that might lead to organ failure and death. Early recognition of symptoms and accurate measurement of core temperature were crucial to rapid diagnosis. Milder forms of heat-related illness are manifested by symptoms such as headache, weakness, dizziness, and an inability to continue activity. These were managed by supportive measures including hydration and moving the patient to a cool place. Hyperthermia and central nervous system symptoms should prompt an evaluation for heat stroke. Initial treatments should focus on lowering core temperature through cold water immersion. Applying ice packs to the head, neck, axilla, and groin is an alternative. Also, the patient must be transported to a cool environment, removing excess clothing, and intravenous hydration. Delayed access to cooling is the leading cause of morbidity and mortality in persons with heat stroke. Identification of at-risk groups can help physicians and community health agencies provide preventive measures.

The present study found significant improvement $(\mathrm{P}=<0.001)$ of correct knowledge about heat exhaustion during pilgrim among nurses at military hospital, the highest improvement was in non-communicable diseases the lowest was in sun stroke prevention. $27 \%$ of nurses had adequate knowledge ( $>60 \%$ from total score) in the pre-test $94 \%$ in the post-test $74 \%$ after 3 month with significant difference among 3 rests regarding adequate knowledge.

Almanza et al. (2007) reported advantage of educational program on the improvement of base line knowledge and attitude toward heat exposure health hazard.

In the present study, there were significant improvement of correct knowledge about hypertension, dengue fever, skin scalding \& others diseases during pilgrim among nurses at military hospital, the highest improvement was in skin scalding prevention the lowest was in first aid bag. $28 \%$ of participant had adequate knowledge ( $>60 \%$ of total score) in the pre-test $92 \%$ in the post-test $61 \%$ after 3 month with significant difference among these tests regarding adequate knowledge. The present study showed that about $89.9 \%$ of individuals interviewed had heard of dengue fever. Itrat et al. (2008) concluded that adult population of Karachi had adequate knowledge related to the disease 'dengue' on isolated aspects, but the overall prevalence of 'sufficient knowledge' based on their criteria was poor. They demonstrated adequate prevalence of preventive practices against dengue was found to be in $38.5 \%$ of the sample, with $66 \%$ of these in Aga-Khan University Hospital and 33\% in Civil Hospital Karachi.

In Egypt, El Bahnasawy et al. (2011) mentioned that geographical expansion of the mosquito has been aided by the international commercial trade particularly in used cartyres which easily accumulate rainwater. Increased air travel and breakdown of vector control measures contributed greatly to the 
global burden of dengue and DH fevers, particularly with the presence of Ae. aegypti in Egypt and endemicity of DF and DHF in the neighboring regional countries mainly Saudi Arabia.

Regarding skin diseases in Saudi Arabia, Bahamdan et al. (1995) analyzed the pattern of skin diseases seen in the dermatology unit of Asir Central Hospital, Abha, Southern KSA, during a 12-month period from March 1992 to March 1993. All new patients who were seen for dematological complaints in the skin clinics, emergency room or in the wards were studied prospectively. A total of 1285 dermatoses were diagnosed in 1223 patients during the 12-month study period. Out of the top 12 group diseases, ecze$\mathrm{ma} /$ dermatitis $(25.68 \%)$ came first, followed by viral infections. Among the eczema group, atopic eczema accounted for $53.64 \%$ and was most frequently observed in children $(110 / 177=62.14 \%)$ compared to adults. The main causes of superficial mycoses were dermatophytosis and candidiasis. Psoriasis was the most common papulosquamous disorder. Scabies was seen in expatriate Egyptians and Asiatics only. Primary pyogenic infections of the skin were uncommon. Shafi et al. (2008) also showed great improvement of knowledge regarding infective skin diseases during pilgrim after complete the intervention program.

Morsy (2012) in Egypt reported that most insect bites cause local inflammatory reactions that subside within a few hours. However, more severe local symptoms, transmission of a disease-causing pathogen, and systemic allergic reactions are also possible. Mosquito bites can cause varying degrees of local swelling, papular urticaria in children, and rare systemic allergic reactions, including anaphylaxis. Papular urticaria is a hypersensitivity reaction most often seen in children following mosquito and flea bites, although a variety of other bites have been implicated in smaller numbers of reports. Systemic allergic reactions can occur in response to the bites of mosquitoes, several types of blood-sucking flies, fleas, kissing bugs, lice, and ticks. Aldridge (2014) stated that psoriasis is a chronic disease that affects more than the skin. It has an impact on every facet of an individual's life and is associated with numerous comorbidities, such as obesity, diabetes, cardiovascular disease, psoriatic arthritis, metabolic syndrome, squamous cell carcinoma, lymphoma, depression, anxiety and other immune-related conditions, such as Crohn's disease. He concluded that with the right education from the community nurse, patients can be informed about the decisions they make and can ultimately choose to live a healthier life.

Tutor evaluation, most adequacy of evaluation was for evaluation of the tutor $(98.0 \%)$ due to good illustration and using of new and interested ways, while least was for program duration $(82.0 \%)$ and most sufficient studied topic was vaccination and the least was meningitis. Guembe et al. (2012) gave information on the impact of care bundles has been mainly acquired in adult intensive care units (ICUs). They found that a single 20-min educational intervention on the prevention of catheter-related bloodstream infection (CRBSI) significantly improved HCWs' knowledge, but was not enough to reduce the incidence density of CRBSI.

Total knowledge Score \& Nurses' sociodemographic characteristics: In the present study nurses in the age group $>30$ year got the highest total knowledge score than others. The graduated nurses have got the highest total knowledge score. Nurses with work experience more than 5 Years have got the highest Total knowledge Score. There was a significant difference between the total knowledge score according to education, and work experience $(\mathrm{P}>0.05)$ in pre and in post and after 3 month in age and in all intervention time in department the highest was ICU then ward then operation room.

Monteverde (2013) stated that the increasing number of ethical issues highlighted in everyday nursing care demonstrates the connectedness between nursing ethics and nurs- 
ing practice. He asked about the role of ethical theories in this context? He added that by making reference to the current and growing global scarcity of nursing care, nursing would no longer be marginalised, but instead at the centre of public health attention and reputation. But, marginalisation persisted by increasingly affecting the care receivers, especially those groups that were pushed to the fringes by consequences of the healthcare market, such as persons of extreme old age, suffering from multiple morbidities, or with poor health literacy. Whereas the classical understanding of the ethics of care focuses on the nurse-patient relationship and on individual care and understanding of ethics, the new understanding confirms the classical, but adds an understanding of social ethics: caring for the access to care is seen as a main ethical goal of social justice within a nursing ethic.

Buxton and Davies (2013) found that the differences with respect to the nutrition knowledge levels between the age groups, gender, work experience and educational background of respondents were not statistically significant at $p<0.05$. They added that findings supported other reports that nurses need more training in nutrition and therefore have important implications for professionals planning curricula for nursing education at the undergraduate level in the university.

Walker (2014) reported that students and staff of all ages look to school nurses as authorities on health and wellness. Health promotion and wellness education are school nursing practices that have the potential for continuous impact on students. School nurses had the critical responsibility of acknowledging their influences on the health behavior of others and are called to take ownership of the essential role of modeling wellness. Chronister (2014) evaluated whether an educational intervention given to $32 \mathrm{RNs}$, increased knowledge and corrected clinical decision making (CDM) for the use of continuous ST-segment monitoring. He found that many RNs responsible for electrocardi- ographic monitoring are not aware of evidence-based ST-segment monitoring practice guidelines and could not properly place precordial leads needed for ST-segment monitoring. Knowledge and correct CDM with ST-segment monitoring could be improved with focused education. Gardner (2014) declared that the nurse and nurse faculty shortage resulted in a continuous demand for new nurse educators. Clinical experts recruited as faculty face numerous challenges in the move to academia. He aimed to understand the lived experience of nurse educators and characteristics, traits, practices, and experiences that influenced their development and competence, by studying a sample of nurse educators with five or more years of full-time teaching experience, peer-nominated as effective teachers, was recruited for phenomenological study. He recommended eight overarching themes including factors to support new faculty entering academia and the process of learning to teach in higher education, and explored characteristics of effective teachers with implications for both new and experienced faculty for attracting more nurses to academia and supporting retention of faculty.

The present study result agreed with Baja et al, (2014) who found that more the age and experience better the knowledge, also this study reported positive effect of educational degree on the level of knowledge and percent of knowledge improvement after educational session held in studied hospitals. But, Gurubacharya et al. (2003) found that knowledge of health care workers about the risk associated with needle-stick injuries and use of preventive measures was inadequate. A standing order procedure (SOP) should be formulated regarding needle-stick injuries in all the health institutions. It should outline precautions to be taken when dealing with blood and body fluids, and contain reporting of all needle-stick injuries. Health care workers should be made aware of hazards, post-exposure prophylaxis to needle-stick injuries and hospital-wide hepatitis immun- 
ization program was a must. Cleary et al. (2013) encouraged higher degree students to develop a strategic to minimize extra demands created by additional studies; and to make most of their work as a student to facilitate development of their careers and establish a track record in doing so. They gave an overview of relevant issues as thesis marking and practicalities discussion.

In Saudi Arabia, Yousuf et al. (2012) conducted a cross-sectional study in Jeddah city using self-administered questionnaire investigated nurse knowledge of breast cancer and practice of early screening in primary health care centres. They found a need to provide continuing education programs to improve nurses' knowledge and practice

In Jordan, Tubaishat et al. (2013) explored nurses' attitudes toward pressure ulcer prevention and to identify the perceived barriers to care in pressure ulcer prevention. They concluded that several barriers need to be resolved first if effective prevention is to be provided. This should form a reference dataset that needs to be addressed in the tissue viability field, which was still in its infancy.

In Iran, Adib-Hajbaghery and Hoseinian (2014) evaluated knowledge, attitude, practice of complementary and traditional medicine by medical staff of Kashan. The questionnaire was consisted of 5 questions regarding demographic characteristics and 12 questions on knowledge, attitude and practice of traditional and complementary medicine. They recommended training especially nurses and doctors on applications, benefits and side effects of complementary and traditional medicine.

\section{Conclusion}

Nurses $(96.0 \%)$ were willing to attend educational program about health hazard during pilgrim mostly to improve practice $(56.0 \%)$ and raise awareness $(44.0 \%)$. The most needed topics were prevention \& vaccination $(26.0 \%)$. There was significant improvement regarding adequate knowledge after program implementation. Evaluation program adequacy the most was tutor $(98 \%)$, while least was program duration $(82 \%)$, and the most sufficient item was vaccination. The education, work experience, age and department were determinant for knowledge level and capacity of education.

\section{Recommendations}

1- To establish a fixed training course for mass gathering infectious diseases at hajj. 2Medical and lab investigation for any suspected infected person who coming after hajj to detect any of the infectious disease that could be introduced to our country. 3Design an efficient plant for early and rapid response to infectious disease disasters.

\section{References}

Aarnio, M, Nieminen, J, Pyörälä, E, Lindblom, S, 2010: Motivating medical students to learn teamwork skills. Med. Teach. 32, 4:e199-204.

Adib-Hajbaghery, M, Hoseinian, M, 2014: Knowledge, attitude and practice toward complementary and traditional medicine among Kashan health care staff, 2012. Complement Ther. Med. 22, 1:126-32.

Afifi, S, Wasfy, MO, Azab, MA, Youssef, FG, Pimentel, G, et al, 2007: Laboratory-based surveillance of patients with bacterial meningitis in Egypt (1998-2004). Euro. J. Clin. Microbiol. Infect. Dis. 26, 5:331-40.

Ahmed, Q, Arabi, M, Memish, Z, 2006: Health risk at the Hajj. Lancet, 25; 9515:1008-15.

Aldridge, A, 2014: The role of the community nurse in psoriatic comorbidities interventions. Br. J. Community Nurs. 19, 1:38-42.

Al-Gahtani, Y, El Bushra, H, Al-Qarawi, S, Al-Zubaidi, A, Fontaine, R, 1995: Epidemiolological investigation of an outbreak of meningococcal meningitis in Makkah (Mecca), Saudi Arabia. Epidemiol. Infect. 115, 3:399-409.

Almanza, BA, Namkung, Y, Ismail, JA, Nelson, D, 2007: Clients' safe food-handling knowledge \& risk behavior in a home-delivered meal program. J. Am. Diet Assoc. 107, 5:816-21.

Almas, K, Al-Malik, TM, Al-Shehri, MA, Skaug, N, 2003: The knowledge and practices of oral hygiene methods and attendance pattern among school teachers in Riyadh, Saudi Arabia. Saudi Med. J. 24, 10:1087-91.

Al-Qurashi, AM, 2006: House dust mites and allergic manifestations among some children in Dammam, Saudi Arabia. J. Egypt. Soc. Parasitol. $36,1: 283-8$. 
Alquthami, AH, Pines, JM, 2014: A systematic review of non-communicable health issues in mass gatherings. Prehosp. Disaster Med. 29, 2:167-75.

Al-Shehri, AM, 2009: Perceptions of primary health care (PHC) doctors on the prevention of coronary heart disease (CHD) in Riyadh, Saudi Arabia. J. Family Commun. Med. 16, 2:49-52.

Al-Shehri, AM, Khoja, TA, 2009: Doctors and leadership of healthcare organizations. Saudi Med. J. 30, 10:1253-5

Al-Tawfiq, JA, Memish, ZA, 2012: The Hajj: updated health hazards and current recommendations for 2012: Euro Surveill. 17, 41:20295

Andow, DA, 2003: Negative and positive data, statistical power, and confidence intervals. Environ. Biosafety Res. 2:75-80.

Bahamdan, KA, Egere, JU, Khare, AK, Tallab, T, Ibrahim, K, et al, 1995: The pattern of skin diseases in Asir region, Saudi Arabia: A 12month prospective study in a referral hospital. Ann. Saudi Med. 15, 5:455-7.

Baja, J, Welker, A, Beck, G, Schleppers, A,Fischer, M, et al, 2014: Professional image of anesthetists in the general public. Influence of provision of information and previous experience with the discipline. Anaesthesist 63, 2:114-21

Balkhy, H, Memish, Z, Bafaqeer, S, Almuneef, M, 2004: Influenza a common viral infection among Hajj pilgrims: time for routine surveillance and vaccination. J. Travel Med. 1, 2:82-6.

Becker, JA, Stewart, LK, 2011: Heat-related illness. Am. Fam. Physician 83, 11:1325-30.

Buxton, C, Davies, A, 2013: Nutritional knowledge levels of nursing students in a tertiary institution: lessons for curriculum planning. Nurse Educ. Pract. 13, 5:355-60.

Chronister, C, 2014: Improving nurses' knowledge of continuous ST-segment monitoring. AACN Adv. Crit. Care 25, 2:104-13.

Chu, DK, Poon, LL, Gomaa, MM, Shehata, MM, Perera, RA, et al, 2014: MERS coronaviruses in dromedary camels, Egypt. Emerg. Infect. Dis. 20, 6:1049-53.

Cleary, M, Happell, B, Walter, G, Hunt, G, 2013: Obtaining higher research degree qualifications: Key strategies to consider. Contemp. Nurse 44, 2:196-203.

El-Bahnasawy, MM, Khalil, HH, Morsy, AT, Morsy, TA, 2011: Threat of dengue fever and dengue haemorrhagic fever to Egypt from travelers. J. Egypt. Soc. Parasitol. 41, 2:289-306.

El-Sayed, A, Prince, A, Fawzy, A, Nadra-Elw- -goud, Abdou, MI, 2013: Sero-prevalence of avian influenza in animals and human in Egypt. Pak. J. Biol. Sci. 16, 11:524-9.

Freedman, DO, Weld, LH, Kozarsky, P, et al, 2006: Spectrum of disease and relation to place of exposure among ill returned travelers. N. Engl. J. Med. 354:119-22.

Freedman, SB, Deiratany, S, Goldman, RD, Benseler, S, 2008: Development of a Caregiver Gastroenteritis Knowledge Questionnaire. Ambul. Pediatr. 8, 4:261-5.

Frenk, J, Chen, L, 2011: Overcoming gaps to advance global health equity: a symposium on new directions for research. Hlth. Res. Policy Syst. Feb 22; 9:11. doi:10.1186/1478-4505-9-11

Gardner, SS, 2014: From learning to teach to teaching effectiveness: nurse educators describe their experiences. Nurs. Educ. Perspect. 35, 2: 106-11.

Gatrad, R, Sheikh, A, 2002: Palliative care for Muslims and issues after death. Int. J. Palliat. Nurs. 8, 12:594-7.

Gurubacharya, DL, Mathura, KC, Karki, D B, 2003: Knowledge, attitude and practices in health care workers on needle-stick injuries. Kathmandu Univ. Med. J. (KUMJ). 1, 2:91-4.

Helminen, $\mathrm{K}$, Tossavainen, $\mathrm{K}$, Turunen, $\mathrm{H}$, 2014: Assessing clinical practice of student nurses: Views of teachers, mentors and students. Nurse Educ. Today 34, 8:1161-6.

Hemida MG, Chu DK, Poon LL, Perera RA, Alhammadi MA, 2914: MERS Coronavirus in Dromedary Camel Herd, Saudi Arabia. Emerg. Infect. Dis. Jul;20(7). doi: 10.3201/eid2007. 140571

Itrat, A, Khan, A, Javaid, S, Kamal, M, Khan, H, et al, 2008: Knowledge, awareness and practices regarding dengue fever among the adult population of dengue hit cosmopolitan. PLoS One. 2008 Jul 9;3(7):e2620. doi: 10.1371 /journal.pone.0002620.

Khalil, HM, Kandeel, HZ, Morsy, AT, 2013: Severe acute respiratory syndrome (SARS) coronavirus: possible re-emergence of the Asianglobal novel threat. J. Egypt. Soc. Parasitol. 43, 2:555-60.

Kravchenko, J, Abernethy, AP, Fawzy, M, Lyerly, HK, 2013: Minimization of heatwave morbidity and mortality. Am. J. Prev. Med. 44, 3:274-82.

Layne, K, Nabeebaccus, A, Fok, H, Lams, B, Thomas, S, et al, 2010: Modernising morning report: innovation in teaching and learning. Clin. 
Teach. 7, 2:77-82.

Madani, TA, Ghabrahm TM, 2007: Meningococcal, influenza virus, and hepatitis B virus vaccination coverage level among health care workers in Hajj. BMC Infect. Dis. 7:80.

Memish, ZA, Almasri, M, Turkestani, A, Al Shangiti, AM, Yezli, S, 2014: Etiology of severe community-acquired pneumonia during Hajj-part of the MERS-CoV surveillance program. Int. J. Infect. Dis. 2014 Jun 23. pii: S1201 -9712(14)01535-5.

Memish, ZA, Goubeaud, A, Bröker, M, Malerczyk, C, Shibl, AM, 2010: Invasive meningococcal disease and travel. J. Infect. Publ. Hlth. 3, 4:143-51.

Memish, ZA, Venkatesh, S, Ahmed, QA, 2003: Travel epidemiology: The Saudi perspective. Int. J. Antimicrob. Agents 21, 2:96-101.

Memish, ZA, Zumla, A, Alhakeem, RF, Assiri, A, Turkestani, A, 2014: Hajj: infectious disease surveillance and control. Lancet. 383, 9934: 2073-82.

Monne, I, Fusaro, A, Al-Blowi, MH, Ismail, MM, Khan, OA, 2008: Co-circulation of two sublineages of HPAI H5N1 virus in the Kingdom of Saudi Arabia with unique molecular signatures suggesting separate introductions into the commercial poultry and falconry sectors. J. Gen. Virol. 89, Pt 11:2691-7.

Monteverde, S, 2013: Nursing ethics and the access to nursing care. Pflege 26, 4:271-80.

Morsy, TA, 2012: Insect bites and what is eating you? J. Egypt. Soc. Parasitol. 42, 2:291-308.

Morsy, TA, 2012: The causes of skin lesions in the returning travelers: with special reference to Egypt. J, Egypt. Soc. Parasitol. 42, 1:135-56.

Paput, A, 2014: Therapeutic education, a factor of compliance and autonomy. Rev. Infirm. 199:24-6

Parant, RL, Pingitore, FR, LaRose, JA, 2014: An Educational Program to Promote Competency in Pediatric Psychiatric Mental Health Nursing. J. Contin. Educ. Nurs. 2014 Jun 20:1-6. doi: 10.3928/00220124-20140620-01.

Parker, A, Swanson, H, Frunchak, V, 2014: Needs of labor and delivery nurses caring for women undergoing pregnancy termination. J. Obstet. Gynecol. Neonatal Nurs. 2014 Jun 30. doi: 10.1111/1552-6909.12475.

Price, B, 2013: Successful preceptorship of newly qualified nurses. Nurs. Stand. 28, 14:51-6. Rajoo, M, Sulicova, A, Mroskova, S, Supinova, M, Plackova, A, 2013: Imported tropical neuroinfections: practice skills and knowledge among nurses and nursing students. Neuro Endocrinol. Lett. 34, 1:S45-7.

Saleh, AM, Ali, HA, Ahmed. SA, Mohammad, NM, Morsy, TA, 2013a: House dust mites: a risk factor to be considered for occupational safety or source of work-related allergens. J. Egypt. Soc. Parasitol. 43, 3:669-78.

Saleh, AM, Ali, HA, Ahmed, SA, Mohammad, NM, Morsy, TA, 2013b: Knowledge and performance regarding management of house dust mites in a military hospital. J. Egypt. Soc. Parasitol. 43, 3:821-40.

Sheahan, SL, Musialowski, R, 2001: Clinical implications of respiratory system changes in aging. J. Gerontol. Nurs. 27, 5:26-39.

Steffen, R, Bouchama, A, Johansson, A, Dvorak, J, Isla, $N$, et al, 2012: Non-communica-ble health risks during mass gatherings. Lancet Infect. Dis. 12, 2:142-9

Tromba, V, Moretti, M, Massaccesi, V, Peparini, I, et al, 2014: Respiratory diseases in pediatric triage: a comparison between a general emergency department and a pediatric emergency department. Pediatr. Emerg. Care 30, 2:81-3.

Tubaishat, A, Aljezawi, M, Al Qadire, M, 2013: Nurses' attitudes and perceived barriers to pressure ulcer prevention in Jordan. J. Wound Care 22, 9:490-7.

Turris, SA, Lund, A, 2012: Triage during mass gatherings. Prehosp. Disaster Med. 27, 6:531-5.

Walker, JR, 2014: Wellness promotion: school nurses as models of health. NASN Sch. Nurse 29, 3:128-9.

Walsh, A, Moore, A, Barber, A, Opsteen, J, 2014: Educational role of nurse practitioners in a family practice centre: Perspectives of learners and nurses. Can. Fam. Physician 60, 6:e316-21.

WHO, 2014: Middle East respiratory syndrome coronavirus (MERS-CoV) summary and literature update - as of 9 May 2014. Geneva: [Accessed 30 May 2014].

Youssef, FG, El-Sakka, H, Azab, A, Eloun, S, Chapman, GD, Ismail, T, et al, 2004: Etiology, antimicrobial susceptibility profiles, and mortality associated with bacterial meningitis among children in Egypt. Ann. Epidemiol. 14, 1:44-8.

Yousuf, SA, Al Amoudi, SM, Nicolas, W, Banjar, HE, Salem, SM, 2012: Do Saudi nurses in primary health care centres have breast cancer knowledge to promote breast cancer awareness? Asian Pac. J. Cancer Prev. 13, 9:4459-64. 